\title{
A l'aurore de la vie
}

Notre planète a un peu plus de quatre milliards d'années et, en à peine un milliard d'années, la vie y apparaissait. La prééminence absolue des conceptions créationnistes explique que peu se soient posés la question des conditions de sa création avant le début du Xxe siècle, avant le soviétique Oparine et l'anglais $\mathrm{Hal}$ dane. Depuis, en revanche, il n'est guère d'énigmes qui aient autant fasciné philosophes et scientifiques, d'où une très abondante bibliographie qui s'est enrichie très récemment de deux ouvrages importants, celui de Christian de Duve [1] et celui d'Antoine Danchin [2]. Ces deux livres, remarquablement documentés, sont les œuvres de biologistes puisant largement dans leur expérience personnelle pour évaluer la crédibilité des différents scenarii proposés, ce qui imprime à ces études des caractères assez différents; Construire une cellule de Christian de Duve, comporte une analyse particulièrement détaillée du contexte énergétique, biochimique et compartimental de l'apparition de la vie alors qu'Une aurore de pierres, d'Antoine Danchin, s'attarde plus sur les aspects informationnels et philosophiques. Par-delà ces différences de tonalité, reflétant la personnalité et le parcours des auteurs, les thèses privilégiées par l'un et par l'autre divergent sur des points importants et se retrouvent sur d'autres, si bien que leur analyse comparée est non seulement passionnante, mais aussi de nature à faire progresser les conceptions des lecteurs auxquels on ne saurait trop conseiller de procéder par eux-mêmes à une telle lecture comparative.

La première partie de l'ouvrage de Christian de Duve est un rappel $m / s n^{\circ} 7$, vol. 7, septembre 91 d'une remarquable concision, sous lequel perce l'érudition, du plan de base de constitution d'une cellule vivante, Antoine Danchin préférant rappeler plus brièvement certains des critères essentiels de la vie et des moments importants de l'évolution des concepts la concernant.

Pour tous deux, les éléments dont disposent les scientifiques pour reconstituer ce qu'ont pu être les débuts de la vie terrestre sont les êtres vivants actuels et leur positionnement dans un arbre évolutif permettant, par extrapolation déductive, de dessiner à grands traits la cellule primitive à l'origine des branches conduisant aux lignées actuelles des archébactéries, des eubactéries et des eucaryotes. L'ancêtre commun, situé au premier embranchement, aurait pu avoir des propriétés voisines d'archébactéries vivant actuellement à haute température dans des sites riches en sulfure d'hydrogène..., conditions peut-être assez proches de celles ayant prévalu sur la terre prébiotique. Beaucoup plus spéculatif est encore l'établissement de la suite d'événements ayant conduit à la constitution de la proto-cellule originelle, car on ne dispose alors, comme éléments d'orientation, que de la composition probable des matériaux terrestres et des conditions possibles régnant durant les quelque six cents millions à un milliard d'années précédant l'apparition de la vie, de quelques résultats expérimentaux permettant de tester la probabilité de certaines hypothèses et de leur vraisemblance en regard de notre connaissance actuelle des mécanismes réactionnels en chimie. En ces temps lointains, donc, la surface terrestre devait être chaude et humide, riche en gaz réducteurs (sulfure d'hydrogène, méthane...) et en oxyde ferreux. L'irradiation solaire intense et des orages violents devaient constituer d'importantes sources d'énergie et de possibles facteurs de déclenchement réactionnel. Toutes ces conditions, comme l'ont bien montré les expériences historiques de Miller en 1953 [3], ont dû permettre la synthèse de molécules organiques, notamment d'acides aminés, de thiols, d'acides dicarboxyliques et, peut-être, quoique plus difficilement, de bases puriques et pyrimidiques. A ce point, existe une importante divergence entre les thèses de De Duve et de Danchin : le premier imagine la suite des réactions abiotiques au sein de la "soupe primitive ", au moins en certains sites privilégiés, alors que le second ne voit dans cette soupe qu'un "brouet insipide " bien incapable d'engendrer les molécules de la vie. La chimie en solution, affirme$t$-il, ne peut favoriser les processus de polymérisation et, de plus, par manque de sélectivité, accumule une multitude de produits de réaction dont la plupart ont toutes les chances de se comporter comme des poisons de chaînes proto-métaboliques qui pourraient s'établir. A la suite de Wächtershäuser [4], il propose que les synthèses prébiotiques aient pu se dérouler à l'interface avec l'eau de supports solides (d'où le titre Une aurore de pierres) comportant des cations métalliques auxquels se seraient fixées par des "pattes" négatives, carboxylates et phosphates, des molécules entrant en réaction. Cette chimie d'interface aurait l'avantage de favoriser la concentration locale des réactifs, les réactions de polymérisation et la sélection des 
produits en fonction de leur charge. En solution, de Duve explique la synthèse des polymères, avant tout des oligopeptides, par la condensation d'acides carboxyliques et de thiols en thioesters, dans des conditions de haute concentration en soufre, de température élevée et de $\mathrm{pH}$ acide. Ces conditions semblent un peu extrêmes, surtout pour ce qui concerne les concentrations des réactifs, qui doivent être molaires, mais pourraient avoir été, de fait, réunies en certains endroits. L'existence des thioesters étant postulée, tout était en place pour le développement d'une riche chimie abiotique, l'équilibre fer ferreux-fer ferrique assurant un bon système de donneurs-accepteurs d'électrons dont l'efficacité a pu, très tôt, être augmentée par l'intervention catalytique de composés fer-soufre. Selon ce schéma, les événements de sélection moléculaire ont pu être, au départ, la solubilité et la stabilité des produits réactionnels, puis la constitution de couples récepteurs-ligands. Cette proposition tout à fait intéressante, et crédible, part de la stabilisation bien connue des protéines par leur ligand et en déduit que, si se sont constitués, dans ce bouillon prébiotique, des oligopeptides ayant une affinité particulière pour un composé réactionnel, ce couple a dû être sélectionné du fait de la stabilisation ainsi engendrée, aboutissant à la première ébauche d'un système enzymesubstrat. L'enchaînement des réactions comportant de telles interactions spécifiques entre des métabolites et des oligopeptides doués d'une activité catalytique rudimentaire a pu conduire à la sélection des premières voies proto-métaboliques.

Christian de Duve ne partage pas l'opinion, largement avancée aujourd'hui, que le "monde de l'ARN ", molécule à la fois informationnelle et catalytique $\left(\mathrm{m} / \mathrm{s}, n^{\circ} 2\right.$, vol. 1, p. 107 ; $n^{\circ}$ 5, vol. 2, p. 280 ; $n^{\circ} 8$, vol. 4, p. 522), a joué un rôle extrêmement précoce dans le développement des constituants de la vie et imagine plutôt la structuration première d'un proto-métabolisme contrôlé par des oligo-catalyseurs oligopeptidiques et des ancêtres des autres soumis à une sorte de sélection darwinienne des "formes complémentaires ", selon le modèle décrit plus haut. L'apparition de l'ARN a pu alors bénéficier de la présence de " proto-enzymes " augmentant la probabilité de la formation d'oligonucléotides dont certaines espèces auraient été rapidement sélectionnées sur la base de leur stabilité (grâce à des appariements internes) et de leur pouvoir autoréplicatif. Ces ancêtres des ARN actuels pouvaient donc avoir beaucoup des caractéristiques des ARN de transfert (petits oligonucléotides largement auto-appariés) et étaient doués d'activité "ribozymatique ", c'est-à-dire catalytique. La formation d'aminoacyl ARN a pu être favorisée par, là encore, la stabilisation ainsi induite de ces deux molécules, ou encore par la plus grande réplicabilité de l'ARN ainsi complexé. Le passage d'une synthèse protéique sans matrice à la traduction d'une information stockée dans des molécules d'ARN "messagers" pourrait, une fois de plus, être le résultat de la sélection successive d'une synthèse peptidique passant par l'intermédiaire des aminoacyl ARN, plus efficace que celle mise en place précédemment, puis de molécules d'ARN permettant de "positionner " correctement les molécules d'aminoacyl ARN, aboutissant à une plus grande reproductibilité et fidélité de la synthèse des espèces " avantagées ". Alternativement, Christian de Duve se hasarde à l'hypothèse iconoclaste selon laquelle les premières proto-enzymes ont pu être recopiées directement en ARN messager par l'intermédiaire des molécules d'aminoacyl ARN les reconnaissant par leur pôle aminoacyl et formant matrice pour le recopiage d'une molécule de messager par leur pôle de l'anticodon. Antoine Danchin, en revanche, se refuse totalement à considérer même l'éventualité de cette " traduction inverse " et est un partisan d'un monde de l'ARN extrêmement précoce, essentiel à l'évolution ultérieure du système, non seulement de par ses propriétés autoréplicatives, catalytiques et rapidement informationnelles, déjà signalées, mais aussi structurales. Il existe dans la vie actuelle quelques exemples de la transformation métabolique d'aminoacides fixés à leur ARN de transfert spécifique dont l'exemple le mieux connu est celui de la synthèse de la sélénocystéine-aminoacyl ARN à partir de la sérine-aminoacyl ARN $(\mathrm{m} / \mathrm{s}$ $n^{\circ}$ 6, vol. 4, p. 392), et Danchin imagine qu'il peut s'agir là des reliques d'un temps ou des proto-ARNt ont pris le relais des pattes acides reliant l'interface réactionnelle à leur support " de pierres ". Cette substitution a pu conduire à l'enchaînement facilité des résidus aminoacyls ainsi fixés, la sélection favorisant peut-être le positionnement correct des proto-ARNt " chargés" par l'intermédiaire de petites molécules d'ARN jouant le double rôle assumé aujourd'hui par les ARN ribosomiques, d'une part, les messagers, d'autre part.

A partir de l'étape maintenant atteinte, les deux auteurs ne divergent guère entre eux ni avec les nombreux autres scénaristes des premiers temps de l'évolution de la vie. La sélection exige la constitution de sous-ensembles, c'est-à-dire le cloisonnement par des membranes, d'abord poreuses, puis beaucoup plus élaborées, comportant des systèmes complexes de récepteurs, de canaux et de transporteurs. Les catalyseurs biologiques et les protéines de structure, maintenant codés par l'information emmagasinée dans de petits messagers, évoluent grâce à une combinatoire de ces proto-messagers, ancêtres probables des exons. Puis, simplement, une dissociation est acquise entre le rôle de stockage de l'information, dévolu à l'ADN, biochimiquement plus stable, et celui d'intermédiaires de l'expression du message, dévolu aux ARN. Nous en sommes alors au stade d'une cellule autonome dont l'information génétique est stockée dans des molécules d'ADN comportant de petites séquences significatives, les exons, séparées par des introns et variablement combinées. La Terre existe depuis moins d'un milliard d'années... il en reste près de trois pour parvenir à la diversité de la vie actuelle. Pour Christian de Duve... et contrairement à la phrase célèbre de Jacques Monod, la Terre était, 
dès l'origine, grosse de la vie, la conjonction des conditions et des éléments originels devant inéluctablement conduire, selon lui, à son éclosion. Et si, ailleurs dans l'univers, de mêmes conditions existent ou ont existé... Antoine Danchin semble avoir une conception bien moins déterministe et, dans la ligne de la pensée de Jacques Monod, considère qu'il a probablement fallu une grande dose de hasard pour que soit réussie, par exemple, la première synthèse de la molécule mère de la vie, l'ARN, et que, toutes choses étant égales par ailleurs, un tel succès peut relever de l'exceptionnel... Mais, une fois atteinte, cette étape oriente précisément la flèche du temps, sans retour en arrière possible, jusqu'à la prochaine bifurcation où, hasard ou nécessité, une direction sera prise, toutes les autres étant définitivement négligées. Quoique les approches de ces deux livres soient manifestement matérialistes, peut-être peut-on remarquer que celle d'Antoine Danchin laisse plus de place à l'intervention éventuelle d'une "volonté " extérieure déterminant les choix faits aux multiples embranchements de l'évolution que celle de Christian de Duve. Les deux ouvrages stimulent, en revanche, identiquement, le rêve - la part d'inconnu demeure suffisante pour ce faire - et suscitent le même émerveillement devant la beauté de l'agencement vital, son incroyable cohérence et ses immenses potentialités évolutives

\section{Axel Kahn}

Ce texte doit également paraître dans La Vie des Sciences, organe d'information de l'Académie des sciences pour lequel il a été écrit.

\section{RÉFÉRENCES}

1. De Duve C. Construire une cellule. Paris : Inter Édition; Bruxelles: De BackWesmael, 1990.

2. Danchin A. Une aurore de pierres. Paris : Le Seuil, 1990.

3. Miller SL, Orgel LE. The origins of life. Englewood Cliffs, NJ, Prentice Hall, 1973. 4. Wächtershäuser G. Before enzymes and templates : theory of surface metabolism. Microbiol Rev 1988.; 52 : 452-84.

$m / s n^{\circ} 7$, vol. 7 , seplembre. 91 